\title{
Flocculation Efficiency of Poly(Acrylamide-Co-Acrylic Acid) Obtained by Electron Beam Irradiation
}

\author{
Gabriela Craciun, ${ }^{1}$ Elena Manaila, ${ }^{1}$ and Maria-Daniela Stelescu ${ }^{2}$ \\ ${ }^{1}$ National Institute for Lasers, Plasma and Radiation Physics, Accelerators Laboratory, 409 Atomistilor Street, \\ 077125 Magurele, Romania \\ ${ }^{2}$ National Research and Development Institute for Textile, 93 Ion Minulescu Street, 031215 Bucharest, Romania \\ Correspondence should be addressed to Gabriela Craciun; gabriela.craciun@inflpr.ro
}

Received 5 December 2012; Revised 14 March 2013; Accepted 22 March 2013

Academic Editor: Maurizio Ferrari

Copyright (C) 2013 Gabriela Craciun et al. This is an open access article distributed under the Creative Commons Attribution License, which permits unrestricted use, distribution, and reproduction in any medium, provided the original work is properly cited.

\begin{abstract}
A correlation between physicochemical characteristics of flocculants obtained by electron beam irradiation and their efficiency for wastewater treatment is presented. For real wastewater treatment, our interest was focused upon total suspended solids, fatty matter, and chemical oxygen demand. Flocculation studies were carried out using a standard jar test. A treatment option based on poly(acrylamide-co-acrylic acid) for wastewater taken from a slaughterhouse plant is presented.
\end{abstract}

\section{Introduction}

Wastewater treatment is a major problem around the world. Growing along with the population growth, industries create environmental problems and health hazards for the population. Hence, environmental concerns behoove scientists and engineers to develop materials and methods to lower the extent of pollution of the environment [1].

Coagulation and flocculation still play a dominant role in many water and Wastewater treatment schemes. Because of the complex interdependence of numerous factors inherent in the coagulation and flocculation processes, a well understanding of the phenomena involved is essential. Coagulation is the process whereby destabilization of given suspension or solution is affected; that is, the function of coagulation is to overcome the factors that promote the stability of a given system. The manifestation of destabilization depends on the particular system; for example, in the case of disperse suspensions or solutions, visible floc or precipitate formation occurs; in the case of highly concentrated suspensions, dewatering of the sludge mass occurs, and so on. Flocculation is the process whereby destabilized particles, or particles formed as a result of destabilization, are induced to come together, make contact and thereby form large( $\mathrm{r}$ ) agglomerates.
In this case, the manifestation of destabilization is realized in practicable terms: in effect, flocculation accelerates floc formation, influences the physical characteristics of flocs formed (e.g., their strength, size, and density), and governs the final concentration of destabilized particles. In water and Wastewater treatment, coagulation and flocculation phenomena are extremely important [2].

In Wastewater treatment, flocculation is essential. Flocculating agents, or flocculants, are important components which cause flocculation, the process of bringing together small particles to form larger particles by adding small quantities of chemicals in water and Wastewater treatment [3, 4]. Flocculants are classified into inorganic and organic categories. Though the inorganic flocculants (also called coagulants) with multivalent metals like aluminum and iron are widely employed, organic flocculants based on acrylamidebased polymers, like polyacrylamide and its derivatives, are generally more effective than their inorganic counterparts as they possess the advantages, such as low dose, ease in handling, no interference with $\mathrm{pH}$ of the suspensions, and larger floc-forming capability $[3,4]$.

Flocculants are applied in potable water, industrial raw and process water, municipal sewage treatment, mineral processing and metallurgy, oil drilling and recovery, and so forth 
[5-11]. These materials have the ability to induce an advanced coagulation process, after which a large amount of bacteria and viruses from the water are precipitated together with the suspended solids of this. Especially flocculants based on poly(acrylamide-co-acrylic acid) obtained by electron beam irradiation are very efficacious for Wastewater treatment. But a strict condition of use is that residual acrylamide monomer content is to be less than $0.05 \%$. The major advantages of radiation induced polymerization processes are (1) very easy to manipulate the molecular weight, from low to very high, by simply changing the feed composition as well as the composition of the product by incorporating different monomers; (2) precise control of charge density as the monomer feed composition is controlled at the initial stages only; (3) precise control of molecular weight distribution; (4) no flammable and toxic solvents used; (5) no production of waste matter or evolution of noxious gases; (6) no production of hazardous effluents; (7) very low monomer contents; (8) very clean process [12].

Physical and chemical properties of flocculants based on poly(acrylamide-co-acrylic acid) obtained by electron beam irradiation are strictly related to the efficiency of Wastewater treatment expressed by the level of treated water quality indicators. There are many situations in which organic flocculants should be used together with classic coagulation aids (inorganic flocculants) such as $\mathrm{Al}_{2}\left(\mathrm{SO}_{4}\right)_{3}, \mathrm{FeSO}_{4}$, or $\mathrm{Ca}(\mathrm{OH})_{2}$ because in the case of very charged Wastewaters, treatments based only on organic flocculants or inorganic flocculants (classical treatment) are less efficient than in the case of their combined use. Moreover, the so-called flocs are larger and more strongly bound than the aggregates obtained by coagulation [1].

It is well known that each Wastewater type, depending on its origin, has its particularities and more than that the same type of Wastewater shows significant variation during the same day. For this reason treatments based on polyelectrolyte should be seen as treatment schemes for each type of Wastewater. These treatment schemes should also be easily adaptable to the significant variations of the same type of Wastewater characteristics. There is no polyelectrolyte with universal destination regardless of the method of production. This is one of the reasons why the field of flocculants obtaining by radiation technologies is still open.

In the present work, we present a treatment option based on poly(acrylamide-co-acrylic acid) for Wastewater taken from a slaughterhouse plant.

\section{Methods}

2.1. Materials and Sample Preparation. For flocculants obtaining, the following materials (from Sigma-Aldrich) were used: acrylamide, AMD (molar mass $71.08 \mathrm{~g} \mathrm{~mol}^{-1}$, density $1.13 \mathrm{~g} / \mathrm{cm}^{3}$, solubility in water $2.04 \mathrm{~kg} / \mathrm{L}$ at $25^{\circ} \mathrm{C}$ ); acrylic acid, AA (molar mass $72.06 \mathrm{~g} \mathrm{~mol}^{-1}$, density $1.051 \mathrm{~g} / \mathrm{mL}$, solubility in water: miscible); sodium chloride, $\mathrm{NaCl}$ (molar mass $58.44 \mathrm{~g} \mathrm{~mol}^{-1}$, density $2.165 \mathrm{~g} / \mathrm{cm}^{3}$, solubility in water: $359 \mathrm{~g} / \mathrm{dm}^{3}$ at $20^{\circ} \mathrm{C}$ ); sodium hydroxide, $\mathrm{NaOH}$ (molar mass $39.99 \mathrm{~g} \mathrm{~mol}^{-1}$, density $2.13 \mathrm{~g} / \mathrm{cm}^{3}$, solubility in water:
TABLE 1: The typical chemical composition of the monomer aqueous solutions.

\begin{tabular}{lc}
\hline Chemical composition & Characteristics \\
\hline Total monomer concentration & $40 \%$ \\
Monomer ration in terms of percentage, & $9 / 1$ \\
$R=$ AMD/AA & $8 \%$ \\
NaCl concentration & $0 \%-0.015 \%$ \\
Initiator concentration, potassium persulfate & \\
Chain transfer agent concentration, sodium & $0.0075 \%$ \\
formate &
\end{tabular}

$1110 \mathrm{~g} / \mathrm{dm}^{3}$ at $20^{\circ} \mathrm{C}$ ); sodium formate, HCOON (molar mass $68.01 \mathrm{~g} \mathrm{~mol}^{-1}$, density $1.92 \mathrm{~g} / \mathrm{cm}^{3}$, solubility in water: $97 \mathrm{~g} / 100 \mathrm{~mL}$ at $20^{\circ} \mathrm{C}$, serves as chain transfer agent in the copolymerization process); potassium persulfate, $\mathrm{K}_{2} \mathrm{~S}_{2} \mathrm{O}_{8}$ (molar mass $270.322 \mathrm{~g} \mathrm{~mol}^{-1}$, density $2.477 \mathrm{~g} / \mathrm{cm}^{3}$, solubility in water: $1.75 \mathrm{~g} / 100 \mathrm{~mL}$ at $0^{\circ} \mathrm{C}$, serves as initiator in the copolymerization process).

For the preparation of acrylamide-acrylic acid monomer solution, 40\% AMD: AA (mass ratio 9:1) and natrium chloride $8 \%$ were dissolved in $1000 \mathrm{~mL}$ distilled water. The optimum $\mathrm{pH}$ monomer solution, which is in the range of 6 to 6.5 in the case of electron beam polymerization, was obtained by gradually adding sodium hydroxide to the monomer solution. From the obtained monomer solution, samples of $100 \mathrm{~mL}$ were distributed in quartz vessels. Different amounts of initiator (potassium persulfate) and chain transfer agent (sodium formate) were added and then each quartz vessel was irradiated with electron beam in atmospheric conditions and at room temperature of $25^{\circ} \mathrm{C}$. The thickness of monomer aqueous solution in each quartz vessel was $23 \mathrm{~mm}$. The typical chemical composition of the monomer aqueous solutions irradiated in order to produce acrylamide-acrylic acid copolymers is presented in Table 1. Electron beam dose rate was fixed at $2.4 \mathrm{kGy} / \mathrm{min}$, in order to accumulate the working dose.

2.2. Experimental Installation. The facility used for poly(acrylamide-co-acrylic acid) flocculants was a linear accelerator called ALIN 10, which is a travelling-wave type, operating at a wavelength of $10 \mathrm{~cm}$ and having $164 \mathrm{~W}$ maximum output power. The accelerating structure is a diskloaded tube operating in the $\pi / 2$ mode. The optimum values of the EB peak current $I_{\mathrm{EB}}$ and $\mathrm{EB}$ energy $E_{\mathrm{EB}}$ to produce maximum output power $P_{\mathrm{EB}}$ for a fixed pulse duration $\tau_{\mathrm{EB}}$ and repetition frequency $f_{\mathrm{EB}}$ are as follows: $E_{\mathrm{EB}}=6.23 \mathrm{MeV}$, $I_{\mathrm{EB}}=75 \mathrm{~mA}$, and $P_{\mathrm{EB}}=164 \mathrm{~W}\left(f_{\mathrm{EB}}=100 \mathrm{~Hz}, \tau_{\mathrm{EB}}=3.5 \mu \mathrm{s}\right)$ [13-15].

\subsection{Poly(Acrylamide-Co-Acrylic Acid) Characterization. EB} irradiation is extremely efficacious in polymerization processes. EB induced polymerization involves the Coulomb interaction of the accelerated electrons with atoms or molecules in the irradiated medium. These interactions generate reactive ions, thermalized electrons, excited states, and radicals to drive the chemical reactions. Polymerization 
is subsequently induced by ionizing radiation in much the same way as free radical addition polymerization. The main function of the ionizing radiation in a radiation-initiated polymerization is limited to the primary events, the initiation step which leads to the production of free radicals and to a few specific secondary effects. The subsequent steps of propagation, termination, and chain transfer proceed as in a chemically catalyzed process [15].

Preparation of poly(acrylamide-co-acrylic acid) flocculants was based on copolymerization by EB irradiation of the aqueous solutions containing appropriate mixtures of acrylamide and acrylic acid monomers and certain agents, such as chain transfer agents to mitigate cross-linking of the polymer structure and initiators to optimize the monomer conversion process. Our interest was focused on the physicochemical properties involved in Wastewater treatment, such as conversion coefficient (CC), residual monomer concentration $\left(M_{r}\right)$, intrinsic viscosity $\left(\eta_{\text {intr }}\right)$ or average $M_{w}$ (molecular weight), and a linearity coefficient given by the Huggins' constant $\left(k_{H}\right)$. These characteristics of poly(acrylamide-co-acrylic acid) obtained by electron beam irradiation technology are influenced by the following factors: chemical composition of the solutions to be irradiated, absorbed dose level, and absorbed dose rate level [15]. Many years of investigations of polymeric material properties [16-19] demonstrated that EB induced polymerization, under proper irradiation conditions and for fixed chemical composition of the monomer mixtures to be irradiated, leads to higher conversion efficiency (near $100 \%$ ) and lower residual monomer concentration (under $0.01 \%)$ than classical polymerization of the acrylamideacrylic acid aqueous solutions.

Two grams of poly(acrylamide-co-acrylic acid) flocculants obtained as above were placed in $200 \mathrm{~mL}$ distilled water for 24 hours and then every sample was stirred for 1 hour at $400 \mathrm{rpm}$ to ensure a very well mixing. The conversion coefficient (CC) and the residual monomer concentration $\left(M_{r}\right)$ were determined based on the bromation reaction of the double-bond method [11,20]. After complete dissolution in water, the copolymer AMD/AA was treated excessively with a bromide-bromate solution and the bromine excess was determined by means of a iodometric method in presence of sodium thiosulfate solution $(1 \mathrm{M})$.

The intrinsic viscosity $\left(\eta_{\text {intr }}\right)$ and the Huggins' constant $\left(k_{H}\right)$ were determined by the viscometry method, using the Hoppler type BH-2 [21]. Hoppler viscometer is a falling ball one. The measured parameter is the time of fall of the ball in a cylindrical tube inclined by $10 \mathrm{deg}$ with respect to the vertical plane and filled with the liquid subject to investigation. The principle of falling-ball viscometers is based on the fact that the viscosity of liquid modifies the speed of ball falling through this liquid. The time of falling through the copolymer solution was measured for five different concentrations of it. As a solvent sodium nitrate $1 \mathrm{~N}\left(\mathrm{NaNO}_{3}\right)$ was used and the working temperature was $30^{\circ} \mathrm{C}$. From the time of falling through the copolymer solution $(t)$ and the time of falling through the solvent $\left(t_{0}\right)$, relative viscosity $\left(\eta_{\text {rel. }}=t / t_{0}\right)$ was obtained. Specific viscosity was calculated from the relation, $\eta_{\text {sp. }}=\eta_{\text {rel. }}-1$. Reduced viscosity was determined using the relation $\eta_{\text {red. }}=\eta_{\text {sp. }} / c$, where $c$ is the copolymer concentration $(\mathrm{g} / \mathrm{dL})$. From the graphical representation of the $\eta_{\text {red. }}$ as a function of the copolymer concentration $(c)$, by extrapolation, the intrinsic viscosity $\eta_{\text {intr. }}$ and $\operatorname{tg} \alpha$ was obtained.

The linearity constant was determined from the following relation: $k_{H}=\operatorname{tg} \alpha /\left[\eta_{\text {intr. }}\right]^{2}$. Intrinsic viscosity is a measure of the hydrodynamic volume occupied by the individual polymer molecules in isolation [22]. In dilute solutions, the polymer chains are separate and the $\left[\eta_{\text {intr. }}\right.$. of a polymer in solution depends only on the dimensions of the polymer chain. Because $\left[\eta_{\text {intr. }}\right]$ indicates the hydrodynamic volume of the polymer molecule and is related to the molecular weight, it provides deep insights on the molecular characteristics [23].

From the Mark-Houwink equation, the relationship among the molecular weight and viscosity is given below: $[\eta]=K *\left[M_{w}{ }^{a}\right]$, where $[\eta]$ is the intrinsic viscosity (dL/g), $M_{w}$ is molecular weight (Dalton), and $K$ and $a$ are constants for a particular polymer-solvent system ( $K$ and $a$ are $3.73 * 10^{-4}$ and 0.66 in $1 \mathrm{~N}$ aqueous sodium nitrate at $30^{\circ} \mathrm{C}$ ) $[21,24]$.

Radius of gyrations is a parameter which characterizes the size of a particle of any shape. The higher the radius of gyration, the more the molecular dimension of the polymer and that will affect the flocculation characteristics synergistically [25]. Radius of gyration was determined by the following relation: $R_{g}=0.0147 * M_{w}{ }^{0.59}[26,27]$. Flocculation studies were carried out on real Wastewater taken from a slaughterhouse plant, at room temperature $\left(20-25^{\circ} \mathrm{C}\right)$ using the standard jar test. The jar-test procedure is commonly used to simulate the water treatment process in the laboratory in order to determine the proper coagulant dosage and accumulate data for process control. Beakers of $1000 \mathrm{~mL}$ capacity each equipped with a variable speed $(0-100 \mathrm{rpm})$ agitator were used. In each beaker, $500 \mathrm{~mL}$ of Wastewater was taken and requisite amount of polymer was added into it [28] by means of a pipette. The agitator was first adjusted to $75 \mathrm{rpm}$ for 2 minutes and then for another 5 minutes at $25 \mathrm{rpm}$. The flocs were then allowed to settle down for 2 minutes [3-19, 28]. A measured volume of samples from each beaker (with and without polymeric flocculant) was taken to determine the suspended solids [29]. It is important that the process using a flocculator involves significant mechanical agitation in order to increase the collision of reagents coagulant and flocculant and the colloidal particles in suspension. However, the cutting action must not be too strong; otherwise, it will destroy the flocs that are formed [30].

2.4. Determination of Wastewater Quality Indicators. The primary purpose of a Wastewater treatment is to remove solids from water. Total suspended solids (TSS) represent the solids which are suspended in Wastewater and would be caught by a filter. Suspended solids are measured by passing Wastewater sample through a filter. The solids caught by the filter, once dried, are the total suspended solids. To measure TSS, the water sample was filtered through a preweighed filter. The residue retained on the filter was dried in an oven at 103 to $105^{\circ} \mathrm{C}$ until the weight of the filter no longer changes. The increase in weight of the filter represents the total suspended 
solids. To calculate the concentration of total suspended solids in the sample the following formula was used:

$$
\operatorname{TSS}(\mathrm{mg} / \mathrm{L})=\frac{(A-B) \times 1000}{V}
$$

where: $A=$ sample and filter weight, $(\mathrm{mg}), B=$ sample and filter weight, (mg), $V$ = volume of water sample, (L).

Determination of extractable compounds with solvents (fatty matter-FM) is a gravimetric method which is used to determine the content of substances that can be extracted with organic solvents from Wastewater and surface water. Solvents used are petroleum ether or hexane or carbontetrachloride. The extractable substances are animal fat and vegetable oil (mineral oil and heavy oil), compounds with functional groups like hydroxyl, carbonyl, carboxyl, organic nitrogen compounds, insecticides, soaps, waxes, resins, and tars that are extracted with solvents. The final result represents the mean value of three determinations made in the same time. The procedure consists of sample acidification to $\mathrm{pH}$ less than or equal to 2 and FM extraction with organic solvent three or four times. The organic extract is evaporated to dryness and the amount of extracted substances is determined by weighing. To calculate the extractable compounds (FM) in the sample, the following formula was used:

$$
\mathrm{FM}(\mathrm{mg} / \mathrm{L})=\frac{m_{2}-m_{1}}{V}-\frac{m_{4}-m_{3}}{V},
$$

where $m_{1}=$ capsule mass with residue from sample, (mg); $m_{2}=$ capsule mass without residue, $(\mathrm{mg}) ; m_{3}=$ capsule mass without residue, blank sample, $(\mathrm{mg}) ; m_{4}=$ capsule mass with residue from blank sample, $(\mathrm{mg})$; and $V=$ volume of water sample, (L).

Determination of organic matter (OM) is an expression of CCOMn consumption. The method is based on permanganate ability to oxidize organic compounds in acid medium if chlorides content is above $300 \mathrm{mg} / \mathrm{L}$. The working procedure is as follows: over the Wastewater sample add $5 \mathrm{~mL}$ of sulfuric acid (diluted 1:2) and $10 \mathrm{~mL}$ potassium permanganate solution $(0.01 \mathrm{~N})$ and then boil for 10 minutes; after that add $10 \mathrm{~mL}$ of oxalic acid $(0.01 \mathrm{~N})$ and titrate with potassium permanganate $(0.01 \mathrm{~N})$ to pale pink lasting 1 minute. The temperature during the titration should by between 50 and $60^{\circ} \mathrm{C}$. To calculate the organic matter in the sample, the following formula was used:

$$
\begin{aligned}
& \mathrm{CCO}-\mathrm{Mn}\left(\mathrm{mg} \mathrm{KMnO}_{4} / \mathrm{L}\right) \\
& =\frac{1000 \times\left[\left(V_{1}+V_{2}\right) \times F-V_{3}\right] \times 0.316}{V},
\end{aligned}
$$

where $V_{1}=$ volume of potassium permanganate solution $0.01 \mathrm{~N}$ initially added in the sample $(10 \mathrm{~mL}) ; V_{2}=$ volume of potassium permanganate solution $0.01 \mathrm{~N}$ used for sample titration $(\mathrm{mL}) ; V_{3}=$ volume of oxalic acid solution $0.01 \mathrm{~N}$ added in the sample $(10 \mathrm{~mL}) ; F=$ factor of potassium permanganate solution $0.01 \mathrm{~N} ; V=$ volume of water sample, $(\mathrm{mL})$.

\section{Results and Discussion}

Wastewater contains solid particles with a wide variety of shapes, sizes, densities, and composition. Specific properties of these particles affect their behaviour in liquid phases and thus the removal capabilities. Many chemical and microbiological contaminants found in Wastewater are adsorbed on or incorporated in the solid particles. Thus, the removal of solid particles and organic matter is essential for purification and recycling of Wastewater [1].

EB irradiation is extremely efficacious in polymerization processes. Polyelectrolytes obtained by electron beam irradiation are branched and exhibit high CC, high $\eta_{\text {intr }}$, and low $M_{r}$, but sometimes low water solubility, especially when $\eta_{\text {intr }}$ is higher than $10 \mathrm{dL} / \mathrm{g}$. In our flocculation studies, we used flocculants $(F)$ having the following physicochemical properties: $C C>95 \%$, a wide variety of $\eta_{\text {intr }}$ between 2 and more than $9 \mathrm{dL} / \mathrm{g}$, small $k_{H}$ values which ensure good water solubility, and $M_{r}<0.05 \%$. Intrinsic viscosity of a polymer is indicative of its hydrodynamic volume in solution, which depends on its molecular weight, structure, and nature of the solvent as well as the temperature of the medium. For two polymers with approximately similar molecular weight, the branched polymer has lower hydrodynamic volume compared to its linear counterpart and thus has lower intrinsic viscosity value. Furthermore, long branches determine higher intrinsic viscosity and vice versa [3]. Intrinsic viscosity of polymers is dependent on their molecular weight and chain dimension $[31,32]$. In dilute solutions, the polymer chains are separate, so intrinsic viscosity of a polymer in solution depends only on the dimensions of the polymer chain and and such the molecular weight $[23,31]$.

In Table 2 the characteristics of five flocculants chosen to be used for Wastewater treatment experiments are presented.

In order to provide measurable improvements in water quality, we have studied the effects of different classical treatments with electrolytes and the effects of various combined treatments with electrolytes and flocculants. Classical treatments (CT) were based on the use of $\mathrm{Al}_{2}\left(\mathrm{SO}_{4}\right)_{3}$ and $\mathrm{CaCO}_{3}$, products that are traditionally used in the coagulationflocculation treatment of wastewaters. For the Wastewater treatment, our interest was focused upon the following quality indicators established by the Romanian Standards concerning the conditions for Wastewater evacuation in the urban sewerage system: total suspended solids (TSS), extractable substances with petroleum ether (fatty matterFM), and chemical oxygen demand by potassium permanganate method (organic matter-OM). Table 3 presents the results of different classical treatments applied on mentioned quality indicators.

For Wastewater treatment, the poly(acrylamide-coacrylic acid) flocculants are used in very small amounts and their application reduces the amount of flocculants necessary in combined treatments in the range of $20 \%-40 \%$. We present below the results of flocculation experiments in which we used the poly(acrylamide-co-acrylic acid) amount of $4 \mathrm{mg} / \mathrm{L}$ together with classic treatment number $2\left(\mathrm{CT}_{2}\right)$. Although classical treatments lead to good results in terms of quality indicators reducing, we decided to use $\mathrm{CT}_{2}$ because 
TABLE 2: The physicochemical properties of flocculants obtained by EB irradiation.

\begin{tabular}{|c|c|c|c|c|c|c|}
\hline \multirow{2}{*}{ Sample } & \multicolumn{6}{|c|}{ Properties of flocculants obtained by EB irradiation } \\
\hline & $\mathrm{CC}(\%)$ & $\eta_{\text {intr }}(\mathrm{dl} / \mathrm{g})$ & $k_{H}$ & $M_{r}(\%)$ & $M_{w}$ & $R_{g}(\mathrm{~nm})$ \\
\hline Flocculant $1\left(F_{1}\right)$ & 100 & 2.77 & 0.31 & 0 & $0.73 \times 10^{6}$ & 43 \\
\hline Flocculant $2\left(F_{2}\right)$ & 97.63 & 4.83 & 0.17 & 0.00426 & $17.01 \times 10^{6}$ & 69 \\
\hline Flocculant $3\left(F_{3}\right)$ & 98.91 & 6.01 & 0.18 & 0.00284 & $23.69 \times 10^{6}$ & 85 \\
\hline Flocculant $4\left(F_{4}\right)$ & 98.66 & 7.94 & 0.24 & 0.00284 & $36.12 \times 10^{6}$ & 109 \\
\hline Flocculant $5\left(F_{5}\right)$ & 96.09 & 9.18 & 0.17 & 0.0426 & $45.01 \times 10^{6}$ & 124 \\
\hline
\end{tabular}

TABLE 3: The quality indicators values after classic treatment applying.

\begin{tabular}{|c|c|c|c|c|c|}
\hline \multicolumn{3}{|c|}{ Classic treatment type (CT) } & \multicolumn{3}{|c|}{ Quality indicators } \\
\hline & $\mathrm{Al}_{2}\left(\mathrm{SO}_{4}\right)_{3}, \mathrm{mg} / \mathrm{dm}^{3}$ & $\mathrm{CaCO}_{3}, \mathrm{mg} / \mathrm{dm}^{3}$ & $\mathrm{TTS}, \mathrm{mg} / \mathrm{dm}^{3}$ & $\mathrm{OM}, \mathrm{mg} \mathrm{KMnO}{ }_{4} / \mathrm{dm}^{3}$ & $\mathrm{FM}, \mathrm{mg} / \mathrm{dm}^{3}$ \\
\hline $\mathrm{CT}_{1}$ & 100 & 100 & 56 & 224.83 & 156 \\
\hline $\mathrm{CT}_{2}$ & 200 & 200 & 74 & 179.03 & 154 \\
\hline $\mathrm{CT}_{3}$ & 300 & 300 & 60 & 137.4 & 84 \\
\hline $\mathrm{CT}_{4}$ & 400 & 400 & 58 & 124.91 & 60 \\
\hline $\mathrm{CT}_{5}$ & 500 & 500 & 60 & 170.71 & 66 \\
\hline $\mathrm{CT}_{6}$ & 600 & 600 & 54 & 133.23 & 44 \\
\hline $\mathrm{CT}_{7}$ & 700 & 700 & 74 & 195.76 & 58 \\
\hline \multicolumn{3}{|c|}{ Raw water } & 676 & 566.2 & 1280 \\
\hline
\end{tabular}

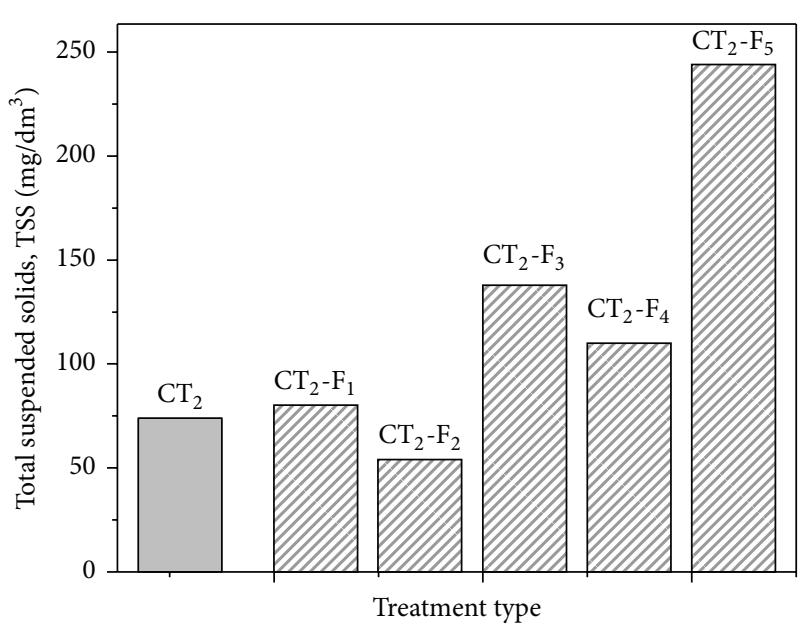

Figure 1: The effect of classic treatment $\mathrm{CT}_{2}$ versus combined treatments $\mathrm{CT}_{2}+\mathrm{F}_{1-5}$ on TSS.

it gave relatively good results and the amount of added aluminum sulphate does not generate what other authors call a secondary pollution [3]. In Figures $1-3$ the variations of treated water quality indicators TSS, OM and FM as a function of the applied treatment $(\mathrm{CT}+F)$ are represented.

Figure 1 shows that combined treatment $\mathrm{CT}_{2}+\mathrm{F}_{2}$ reduce TSS below the level obtained by applying classical treatment $\mathrm{CT}_{2}$ only. Flocculant number 2 is a modest one from an intrinsic viscosity point of view, and the result of its use in the combined treatment suggests that to reduce TSS, a high intrinsic viscosity is not required.

In the case of relatively charged Wastewaters, it is difficult to reduce OM quality indicator. This can be seen from the

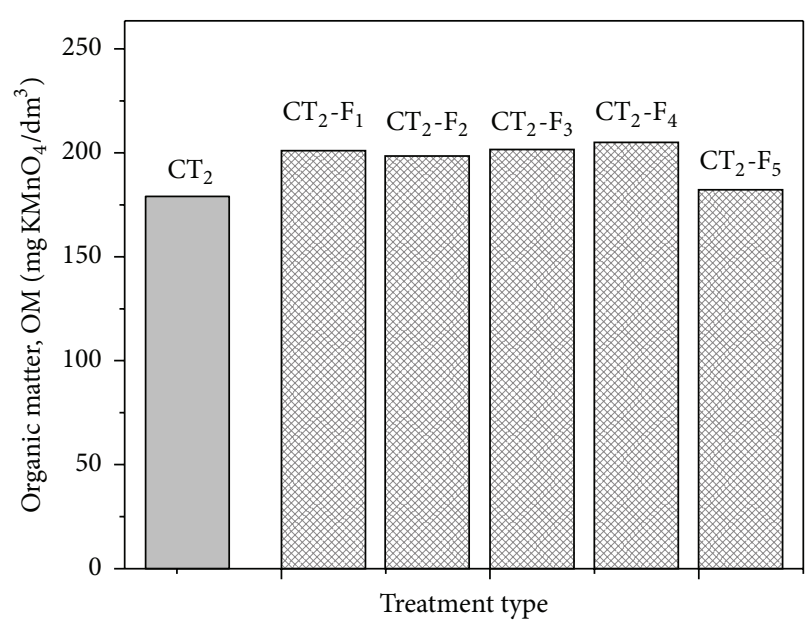

Figure 2: The effect of classic treatment $\mathrm{CT}_{2}$ versus combined treatments $\mathrm{CT}_{2}+\mathrm{F}_{1-5}$ on OM.

results presented in Figure 2 which show that both classical treatment and all combined treatments gave modest results. However, the combined treatments $\mathrm{CT}_{2}+F_{5}$ caused an $\mathrm{OM}$ reduction slightly below the one obtained by applying classical treatment $\mathrm{CT}_{2}$ only. Flocculant number 5 has the highest intrinsic viscosity of all those used in these flocculation experiments. The result obtained after its use as a flocculation adjuvant leads to the idea that flocculant should have a high intrinsic viscosity.

Quality indicator FM is the most difficult to be reduced from all Wastewater quality indicators. All flocculants used in combination with $\mathrm{CT}_{2}$ greatly reduced this quality indicator, but the best results were obtained from the use of the 


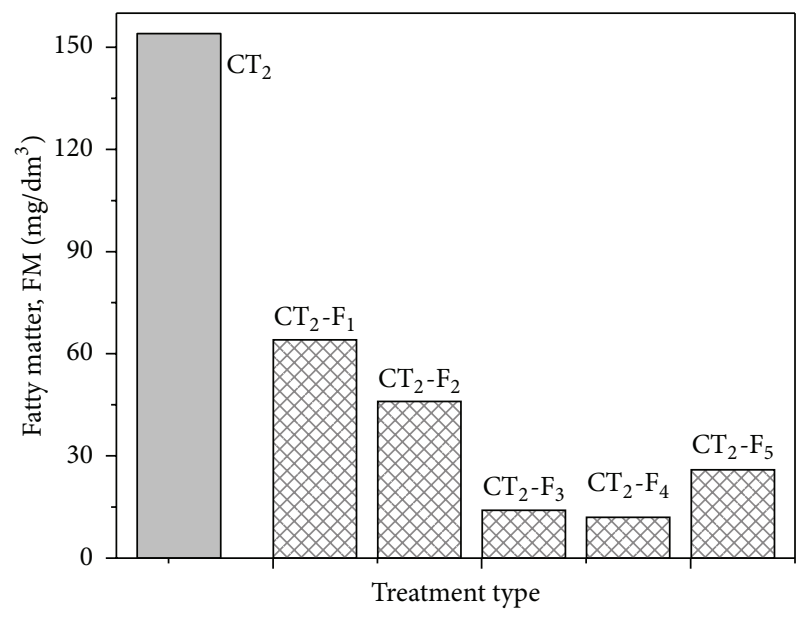

FIgURE 3: The effect of classic treatment $\mathrm{CT}_{2}$ versus combined treatments $\mathrm{CT}_{2}+\mathrm{F}_{1-5}$ on FM.

combined treatment $\mathrm{CT}_{2}-F_{3}$ and $\mathrm{CT}_{2}-F_{4}$. The reduction was almost $90 \%$ (87\% and $89 \%$, resp.). Flocculants $F_{3}$ and $F_{4}$ have high intrinsic viscosities but not the highest. If the intrinsic viscosity increases, we observe a downward trend in terms of FM reduction efficiency.

\section{Conclusions}

Poly(acrylamide-co-acrylic acid) flocculants obtained by EB irradiation have the capability to provide measurable improvements in Wastewater quality, especially leading to a significant reduction of the FM quality indicator (the most difficult to be reduced), compared with the classical treatments. Are used in very small amounts and their application reduces the necessary amount of classical electrolytes. So, these products exhibit good performance in Wastewater treatment and eliminate shortcomings such as secondary pollution of classical treatments.

\section{References}

[1] W. Brostow, S. Pal, and R. P. Singh, "A model of flocculation," Materials Letters, vol. 61, no. 22, pp. 4381-4384, 2007.

[2] J. Bratby, "Introduction," in Coagulation and Flocculation in Water and WasteWater Treatment, chapter 1, pp. 1-8, IWA, London, UK, 2006.

[3] C. Xie, Y. Feng, W. Cao, H. Teng, J. Li, and Z. Lu, "Novel biodegradable flocculating agents prepared by grafting polyacrylamide to konjac," Journal of Applied Polymer Science, vol. 111, no. 5, pp. 2527-2536, 2009.

[4] J. Bratby, "Testing and control of coagulation and flocculation," in Coagulation and Flocculation in Water and Wastewater Treatment, chapter 8, pp. 279-290, IWA, London, UK, 2006.

[5] J. K. Edzwald, "Coagulation in drinking water treatment: particles, organics and coagulants," Water Science and Technology, vol. 27, no. 11, pp. 21-35, 1993.

[6] J. Fettig, H. Ratnaweera, and H. Odegaard, "Synthetic organic polymers as primary coagulants in wastewater treatment," Water Supply, vol. 9, no. 1, pp. 19-26, 1991.
[7] C. L. McCormick, R. D. Hester, S. E. Morgan, and A. M. Safieddine, "Water-soluble copolymers. 31. Effects of molecular parameters, solvation, and polymer associations on drag reduction performance," Macromolecules, vol. 23, no. 8, pp. 2132-2139, 1990.

[8] C. L. McCormick, R. D. Hester, S. E. Morgan, and A. M. Safieddine, "Water-soluble copolymers. 30. Effects of molecular structure on drag reduction efficiency," Macromolecules, vol. 23, no. 8, pp. 2124-2131, 1990.

[9] C. L. McCormick, S. E. Morgan, and R. D. Hester, “The roles of molecular structure and solvation on drag reduction in aqueous solutions," in Water Soluble Polymers: Synthesis, Solutions Properties and Applications, vol. 467 of ACS Symposium, chapter 21, pp. 320-327, American Chemical Society, Washington, DC, USA, 1991.

[10] N. Narkis, B. Ghattas, M. Rebhun, and A. J. Rubin, "The mechanism of flocculation with aluminium sALts in combination with polymeric flocculants as flocculant aids," Water Supply, vol. 9, no. 1, pp. 37-44, 1991.

[11] P. Selvapathy and M. J. Reddy, "Effect of polyelectrolytes on turbidity removal," Water Supply, vol. 10, no. 4, pp. 175-178, 1992.

[12] D. Martin, G. Craciun, E. Manaila et al., "Waste treatment by microwave and electron," in Proceedings of the 2nd Environmental Physics Conference, pp. 91-100, 2006.

[13] D. I. Martin, D. I. Ighigeanu, E. N. Mateescu et al., "Combined Microwave and Accelerated Electron Beam Irradiation Facilities for Applied Physics and Chemistry," IEEE Transactions on Industry Applications, vol. 40, no. 1, pp. 41-52, 2004.

[14] D. I. Martin, E. Mateescu, G. Craciun, D. Ighigeanu, and A. Ighigeanu, "Polymeric flocculants processing by accelerated electron beams and microwave heating," Radiation Physics and Chemistry, vol. 64, no. 5-6, pp. 423-428, 2002.

[15] E. Manaila, D. Martin, G. Craciun, D. Ighigeanu, C. Oproiu, and N. Iacob, "IAEA-CN-115-49 Electron beam processed polyelectrolytes," in Proceedings of International Symposium on Utilization of Accelerators (CD-ROM), pp. 1-8, Dubrovnik, Croatia, 2005.

[16] D. Martin, M. Fiti, L. A. Radu et al., "Low power-high energy linacs for irradiation in polymeric systems," Radiation Physics and Chemistry, vol. 45, no. 4, pp. 615-621, 1995.

[17] D. Martin, M. Dragusin, A. Radu et al., "IAP linacs in applied research," Nuclear Instruments and Methods in Physics Research B, vol. 113, no. 1-4, pp. 106-109, 1996.

[18] D. Martin, M. Dragusin, M. Radoiu et al., "Polymers for waste water treatment," Progress in Colloid and Polymer Science, vol. 102, pp. 147-151, 1996.

[19] D. Martin, M. Radoiu, I. Calinescu et al., "Combined electron beam and microwave treatment for flue gas purification," Materials and Manufacturing Processes, vol. 14, no. 3, pp. 365382, 1999.

[20] M. J. Caulfield, X. Hao, G. G. Qiao, and D. H. Solomon, "Degradation on polyacrylamides. Part II. Polyacrylamide gels," Polymer, vol. 44, no. 14, pp. 3817-3826, 2003.

[21] M. Dimonie, C. Boghina, C. Cincu, M. Marinescu, and N. Marinescu, Poliacrilamida, Editura Tehnica, Bucuresti, Romania, 1986.

[22] R. K. Richardson and S. Kasapis, "Rheological methods in the characterisation of food biopolymers," Developments in Food Science C, vol. 39, pp. 1-48, 1998.

[23] M. A. Rao, "Introduction," in Rheology of Fluid and Semisolid Foods, pp. 1-24, Aspen Publishers, Gaithersburg, Md, USA, 1999. 
[24] M. E. Zeynali and A. Rabbii, "Alkaline hydrolysis of polyacrylamide and study on poly(acrylamide-co-sodium acrylate) properties," Iranian Polymer Journal, vol. 11, no. 4, pp. 269-275, 2002.

[25] S. Pal, D. Mal, and R. P. Singh, "Synthesis and characterization of cationic guar gum: a high performance flocculating agent," Journal of Applied Polymer Science, vol. 105, no. 6, pp. 32403245, 2007.

[26] R. L. Shogren, "Flocculation of kaolin by waxy maize starch phosphates," Carbohydrate Polymers, vol. 76, no. 4, pp. 639-644, 2009.

[27] P. M. Patterson and A. M. Jamieson, "Molecular weight scaling of the transport properties of polyacrylamide in water," Macromolecules, vol. 18, no. 2, pp. 266-272, 1985.

[28] S. Pal, D. Mal, and R. P. Singh, "Cationic starch: an effective flocculating agent," Carbohydrate Polymers, vol. 59, no. 4, pp. 417-423, 2005.

[29] A. Jha, S. Agrawal, A. Mishra, and J. P. Rai, "Synthesis, characterization and flocculation efficiency of poly(acrylamide-coacrylic acid) in tannery waste-water," Iranian Polymer Journal, vol. 10, no. 2, pp. 85-90, 2001.

[30] Y.-H. Bae, H.-J. Kim, E.-J. Lee, N.-C. Sung, S.-S. Lee, and Y.H. Kim, "Potable water treatment by polyacrylamide base flocculants coupled with an inorganic coagulant," Environmental Engineering Research, vol. 12, no. 1, pp. 21-29, 2007.

[31] H. A. Khouryieh, T. J. Herald, F. Aramouni, and S. Alavi, "Intrinsic viscosity and viscoelastic properties of xanthan/guar mixtures in dilute solutions: effect of salt concentration on the polymer interactions," Food Research International, vol. 40, no. 7, pp. 883-893, 2007.

[32] P. J. Flory, "The structure of vinyl polymers," in Principles of Polymer Chemistry, chapter 6, pp. 308-314, Cornell University Press, New York, NY, USA, 1953. 

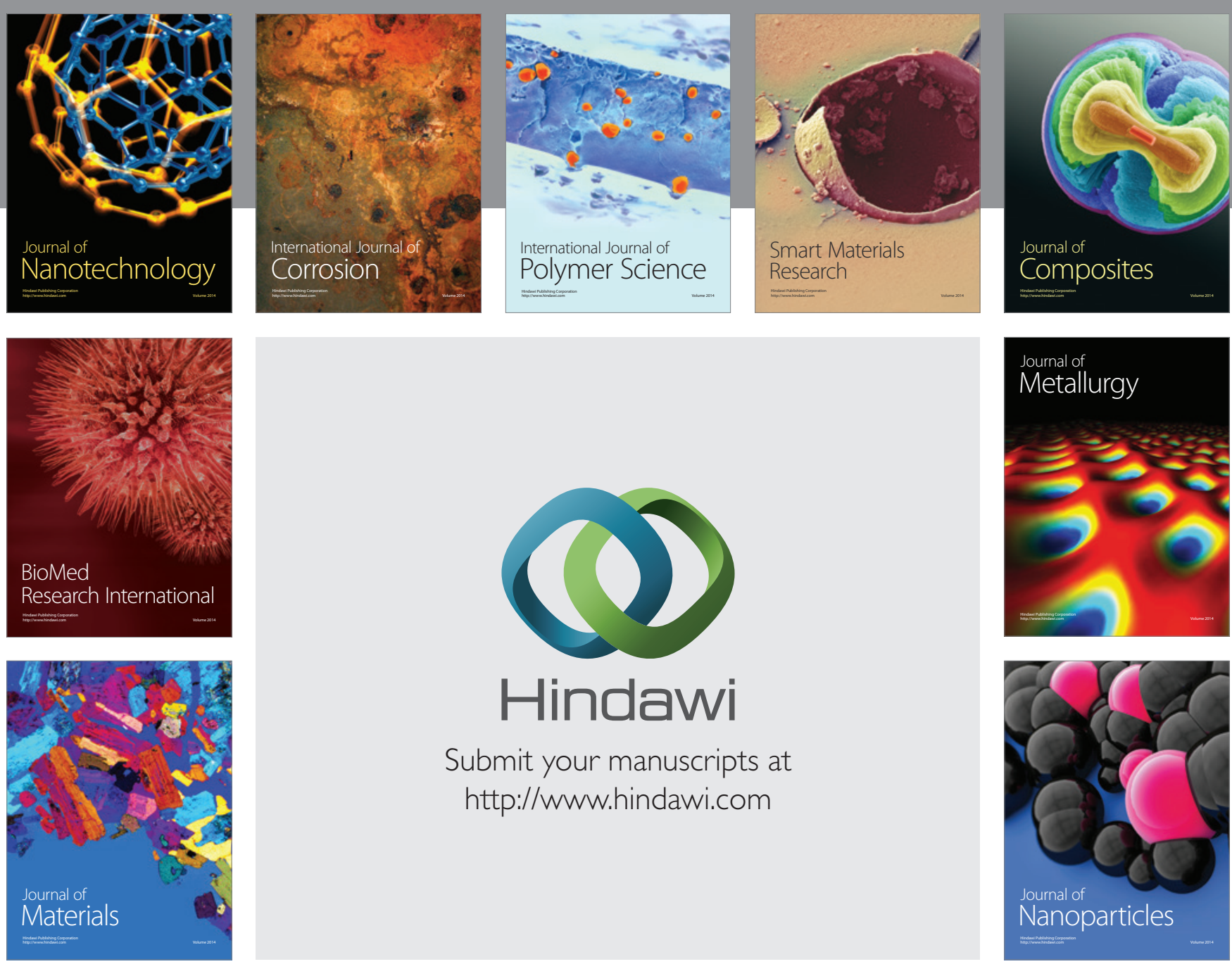

Submit your manuscripts at http://www.hindawi.com
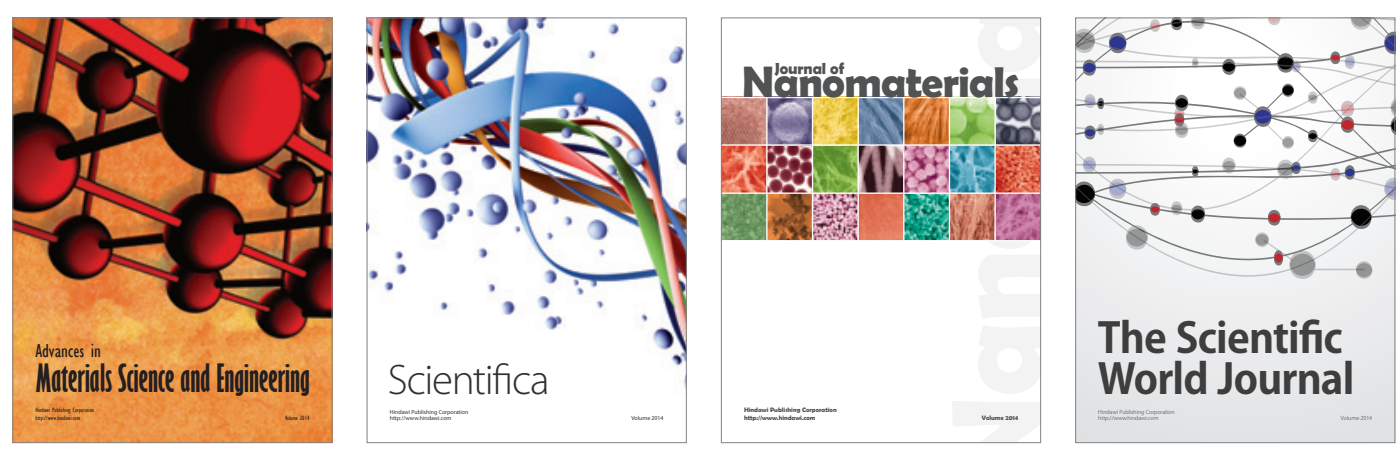

\section{The Scientific World Journal}
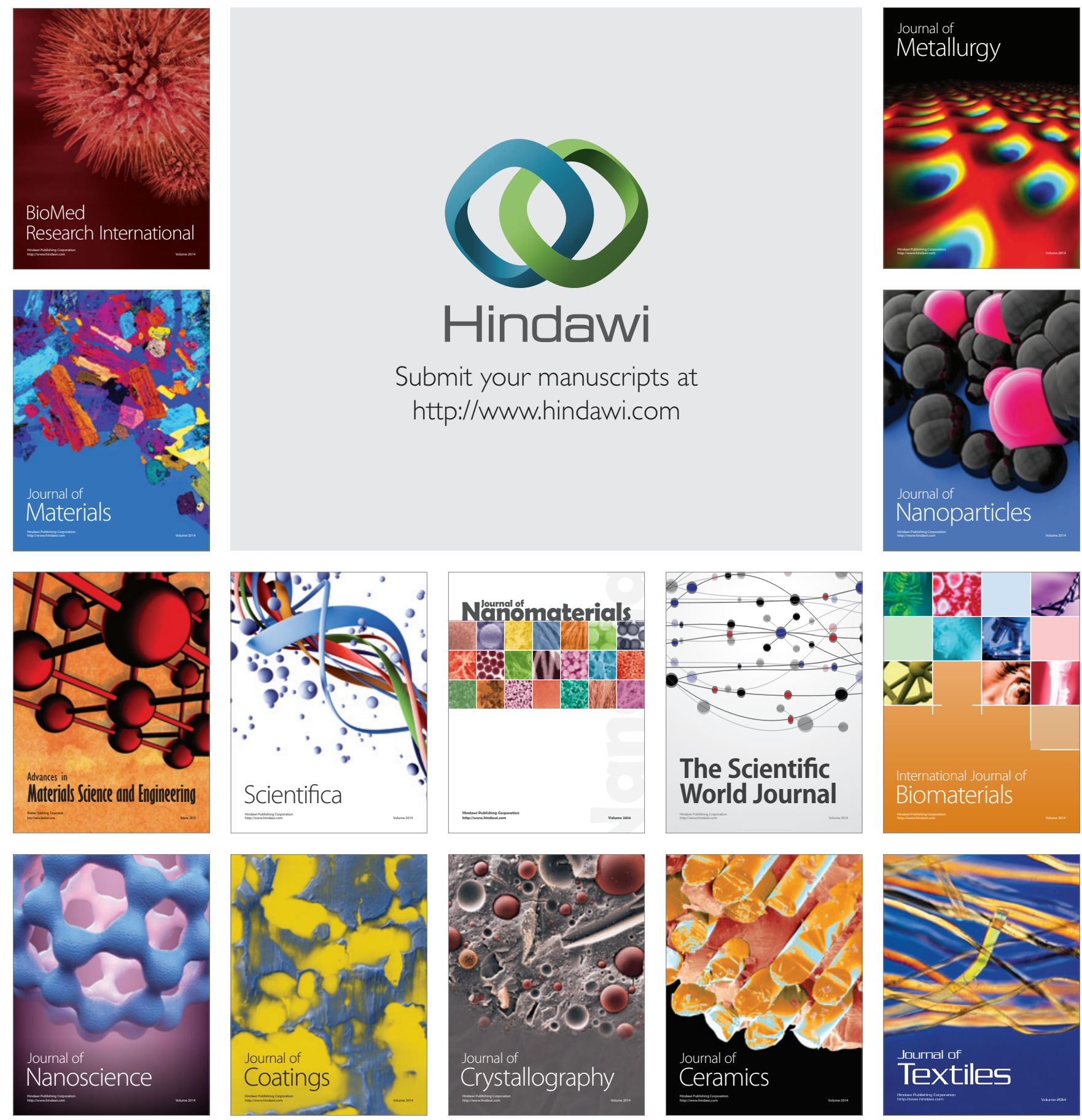